\title{
Workshop on Web APIs and Service Architecture (WS-REST) Chairs' Welcome
}

Welcome to the 9th International Workshop on Web APIs and Service Architecture (WS-REST). First held in 2010 at WWW in Raleigh, North Carolina, USA, this 2018 edition of the WS-REST Workshop Series is proud to be a part of the renowned WWW conference series in Lyon, France. WS-REST 2018 brings together a community of researcher and practitioners interested in Web APIs and service architecture.

\section{Bringing Research and Industry Together}

In keeping with the history of WS-REST events, the 2018 edition strives to bring together vital content from both the Web Services and REST communities. This year we are hosting two individual tracks (Research and Industry) as a way to continue and strengthen this collaboration between academic and applied experience.

The research track submissions received careful peer-review and will be published in the Web Conference proceedings. Industry track submissions focus on field-tested examples and use-cases in the form of extended abstracts or position papers and were selected by the organizers with advice from select program committee members.

\section{APIs, Services, and REST}

APIs have become the connective fabric of the Web and any application area that uses Internet or Web technologies. The goal of WS-REST 2018 is to provide a forum for researchers and practitioners where they can openly and freely exchange ideas about how they are using Web technologies in their APIs, what works and what does not work for them, and what challenges they see in the current landscape of standards and technologies. Our goal is to capture both the state of the art when it comes to Web APIs and service architecture, but to also provide a forum that identifies some of the most pressing issues in that space, and can help solving them.

\author{
Erik Widle \\ Workshop Chair \\ Mike Amundsen \\ API Academy at CA \\ Workshop Chair \\ Technologies \\ API Academy at CA \\ Technologies \\ Mehdi Medjaoui \\ Workshop Chair \\ API Academy at CA Technologies
}

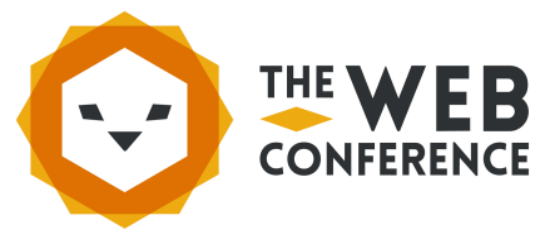




\section{Workshop on Web APIs and Service Architecture (WS-REST) Organization}

Workshop Chairs: Erik Widle, API Academy at CA Technologies

Mike Amundsen, API Academy at CA Technologies

Mehdi Medjaoui, API Academy at CA Technologies

Program Committee: Pierre-Antoine Champin, Université Claude Bernard Lyon 1

Dan Ciruli, Google

Philipp Leitner, Chalmers University of Technology

Simon Mayer, TU Graz

Nandana Mihindukulasooriya, Universidad Politécnica de Madrid

Michael Mrissa, Université de Pau et des Pays de l'Adour

Irakli Nadareishvili, Capital One

Cesare Pautasso, University of Lugano

Silvia Schreier, METRO

Thomas Steiner, Google

Vlad Stirbu, Nokia

Herbert Van De Sompel, Los Alamos National Laboratory

Ruben Verborgh, Ghent University

Erik Wittern, IBM Research

Olaf Zimmermann, University for Applied Science Rapperswil 